\title{
Are Concerns Over Right Laparoscopic Donor Nephrectomy Unwarranted?
}

Joseph F. Buell, MD, * Michael Edye, MD, † Mark Johnson, MD, $\ddagger$ Christine Li, MD,§ Alan Koffron, MD, ,| Eugene Cho, MD, $\S$

Paul Kuo, MD, ๆ Lynt Johnson, MD, ๆ Michael Hanaway, MD, ${ }^{*}$ Steven R. Potter,MD, * David S. Bruce, MD,\#

David C. Cronin, MD, PhD,\# Kenneth A. Newell, MD, PhD,\# Joseph Leventhal, MD,\| Stephen Jacobs, MD,§

E. Steve Woodle, MD, ${ }^{*}$ Stephen T. Bartlett, MD, $\S$ and John L. Flowers, MD§

From the Departments of Surgery, *University of Cincinnati, Cincinnati, Ohio; †New York University, New York, New York; ‡University of North Carolina, Chapel Hill, North Carolina; §University of Maryland, Baltimore, Maryland; |Northwestern University, Chicago, Illinois; MGeorgetown University, Washington DC; and \#University of Chicago, Chicago, Illinois

\section{Objective}

To examine the ability of several large, experienced transplantation centers to perform right-sided laparoscopic donor nephrectomy safely with equivalent long-term renal allograft function.

\section{Summary Background Data}

Early reports noted a higher incidence of renal vein thrombosis and eventual graft loss. However, exclusion of right-sided donors would deprive a significant proportion of donors a laparoscopically harvested graft.

\section{Methods}

A retrospective review was performed among 97 patients from seven centers performing right-sided laparoscopic donor nephrectomy. Surgical and postoperative demographic factors were evaluated. Complications were identified and longterm renal allograft function was compared with historical leftsided laparoscopic donor nephrectomy cohorts.

\section{Results}

Right laparoscopic donor nephrectomy was performed for varying reasons, including multiple left renal arteries or veins, smaller right kidney, or cystic right renal mass. Mean surgical time was $235.0 \pm 66.7$ minutes, with a mean blood loss of $139 \pm 165.8 \mathrm{~mL}$. Conversion was required in three patients secondary to bleeding or anatomical anomalies. Mean warm ischemic time was limited at $238 \pm 112$ seconds. Return to diet was achieved on average after $7.5 \pm 2.3$ hours, with mean discharge at $54.6 \pm 22.8$ hours. Two grafts were lost during the early experience of these centers to renal vein thrombosis. Both surgical and postoperative complications were limited, with few long-term adverse effects. Mean serum creatinine levels were higher than open and left laparoscopic donor nephrectomy on postoperative day 1 , but at all remaining intervals the right laparoscopic donors had equivalent creatinine values.

\section{Conclusions}

These results confirm that right laparoscopic donor nephrectomy provides similar patient benefits, including early return to diet and discharge. Long-term creatinine values were no higher than in traditional open donor or left laparoscopic donor cohorts. These results establish that early concerns about high thrombosis rates are not supported by a multiinstitutional review of laparoscopic right donor nephrectomies.
Laparoscopic donor nephrectomy has been a revolutionary approach used to address the increasing disparity between organ need and availability. ${ }^{1}$ Multiple studies have shown that when cadaveric kidney transplantation is compared with live-donor transplantation, superior graft func-

Presented at the 112th Annual Meeting of the Southern Surgical Association, December 4-6, 2000, Palm Beach, Florida.

Correspondence: Joseph F. Buell, MD, Division of Transplantation, Dept. of Surgery, 230 Bethesda Ave., Cincinnati, OH 45267-0558.

Accepted for publication December 2000. tion and graft survival are noted in the live-donor group., ${ }^{2,3}$ Recent data support that laparoscopic donor nephrectomy increases the rate of donorship while preserving renal graft function. ${ }^{1}$ Left donor nephrectomy has been accepted as the preferential organ for live-donor nephrectomy because of the resulting longer renal vein. ${ }^{4-7}$ Right donor nephrectomy is reserved for instances when the left kidney is determined to be unacceptable for transplantation. Indications most often cited are multiple left renal arteries or veins, anomalous left anatomy, smaller right kidney, or a cystic mass in the right kidney. 8,9 


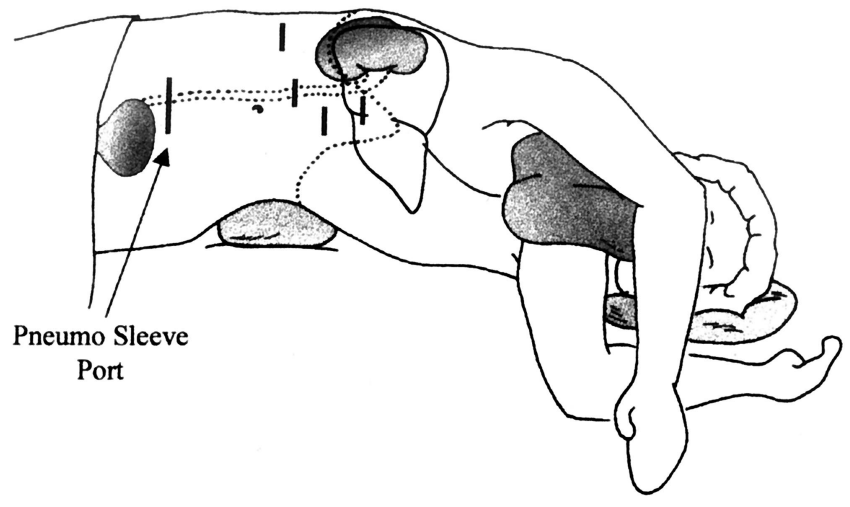

Figure 1. Patient positioning in the left lateral decubitus position, with port placements for the camera, working ports and liver retractor.

Early experiences from Johns Hopkins found an increased incidence of venous thrombosis with eventual graft loss when performing right laparoscopic donor nephrectomy. ${ }^{8}$ The Johns Hopkins group advocated several changes in surgical approach, including relocation of the extraction port and stapling port and open division of the renal vein. However, the conclusion of this study advocated right laparoscopic donor nephrectomy with hesitation and stated that a "rational approach to both donor and recipient operation is crucial." We undertook the current study to examine the varying experiences of other centers well versed in laparoscopic donor nephrectomy to discern whether the Johns Hopkins experience is a universal phenomenon or is an isolated point on a steep learning curve.

\section{METHODS}

This study involved a retrospective analysis of 97 patients identified by seven centers who underwent attempted rightsided laparoscopic donor nephrectomy from January 1997 to October 2000. The centers contributing patients were the University of Cincinnati, New York University, University of Maryland, Northwestern University, University of North Carolina, Georgetown University, and University of Chicago. Reasons for selection of the right kidney included multiple vascular vessels and anomalies of either the left renal artery or vein, a smaller right kidney, or a cystic mass involving the right kidney. All candidates underwent some form of preoperative imaging (spiral computed tomography with three-dimensional reconstruction, magnetic resonance imaging, or angiography, depending on each surgical group's preference and experience). Both traditional laparoscopic techniques, as described by the University of Maryland and Johns Hopkins groups, and the application of the hand-assisted laparoscopy, as described by the University of Michigan and Chicago groups, were used. The use of systemic versus back-table heparinization varied between groups.

The surgical procedures were performed with the patient in the left lateral decubitus position (Fig. 1). Either three or four ports were placed for the laparoscopic and surgical instruments (Fig. 2). The most cephalad port was either 5 or $10 \mathrm{~mm}$, depending on the surgeon's preference of laparoscopic optics. The second port was $12 \mathrm{~mm}$ to allow placement of the endovascular stapler. The most lateral port was $5 \mathrm{~mm}$, which can admit a small harmonic scalpel. When used, the fourth port was $5 \mathrm{~mm}$ and was placed medially for retraction of the right lobe of the liver (Fig. 3). Full mobilization of the vena cava was performed by medial rotation of the duodenum and dissection superiorly and inferiorly to the renal vein while the right lobe of the liver was retracted superiorly. Once the cava was mobilized, the right artery was identified from the anterior aspect. The kidney was then mobilized from the retroperitoneum and flipped forward to allow the posterior aspect of the renal artery to be exposed. After completion of circumferential dissection and mobilization of the renal vessels, the kidney was retracted laterally using either endoscopic kittners or with the attending's hand placed through the hand-assist port, where the kidney can be retracted for the surgeon. This method allows the renal vein to be extended and a small portion of vena cava to be incorporated into the vascular staple line. Extraction was accomplished by multiple methods, including direct hand or extraction bag technique by a midline open incision, a Pfannenstiel incision, or a right lower quadrant incision with placement of a hand-assist device. Once removed, the vena cava was meticulously inspected for bleeding.

Patient demographics collected included sex, age, weight, relation to recipient, and indication for right-sided laparoscopic donor nephrectomy. Surgical demographics included surgical times, rate and indication for conversion, number of renal arteries encountered, estimated blood loss, and warm ischemic times. The incidence of intraoperative and postoperative complications was identified and reviewed. Postoperative parameters included time to regular diet, need for parenteral narcotics, length of hospital stay, and complications (Figs 4 and 5). Long-term assessment of renal allograft

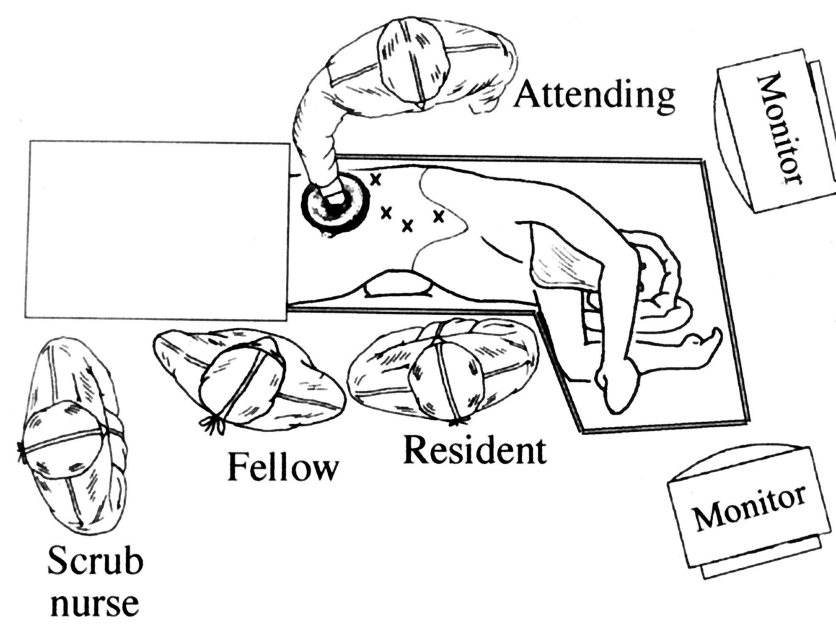

Figure 2. Placement of the surgical team, monitors, and the port and hand-assist device. 


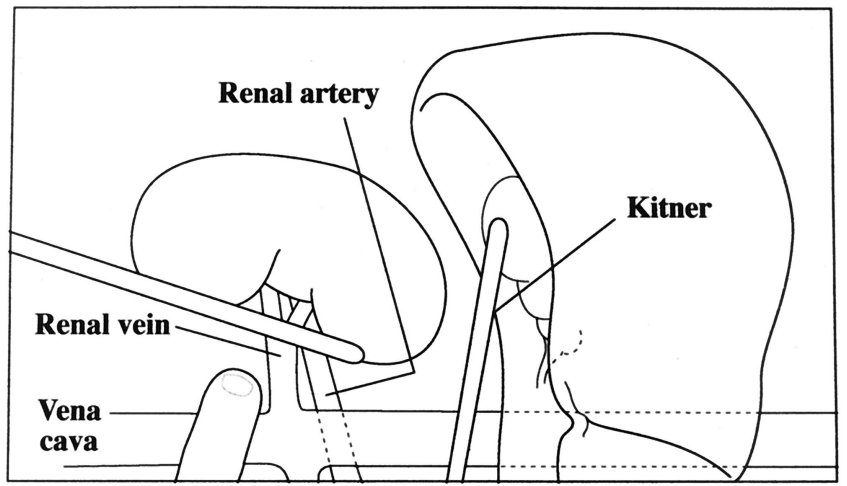

Figure 3. Kidney mobilization with retraction of the liver.

function was accomplished by comparing right-sided donor nephrectomy creatinine values with previously reported open and left laparoscopic donor creatinine values from the University of Maryland at 1, 7, 30, 60, 90, 180, and 365 days. Comparisons were performed using the Fisher exact test; statistical significance was set at $P<.05$.

\section{RESULTS}

Right-sided laparoscopic donor nephrectomy was indicated for varying reasons: multiple renal arteries $(n=59)$, renal cysts $(\mathrm{n}=8)$, small right kidney $(\mathrm{n}=5)$, retroaortic renal vein $(n=6)$, renal artery stenosis $(n=1)$, early left renal artery bifurcation $(n=2)$, ectopic kidney $(n=1)$, and unknown $(\mathrm{n}=14)$ (Table 1). The sex distribution of donors was almost identical (52 women, 45 men) (Table 2). Most of the donor-recipient pairs were living related (73/97; $73 \%$ ). The mean donor age was $40.3 \pm 12.5$ years (range $18-69$ ). The mean donor weight was $171.0 \pm 37.1$ pounds (range 110-280). Arterial anatomy was elucidated by aortography with selective renal injections in most patients (51/97; 51\%), followed by spiral computed tomography $(32 / 97 ; 32 \%)$ and then magnetic resonance imaging (12/97;

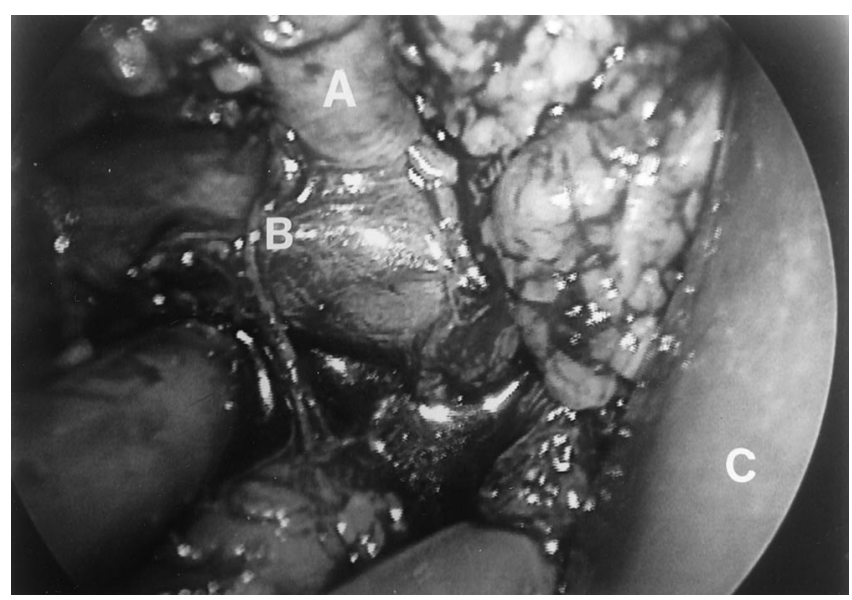

Figure 4. Mobilization of the vena cava with the surgeon's hand inferiorly exposing the renal artery (A), vena cava (B), and liver (C).

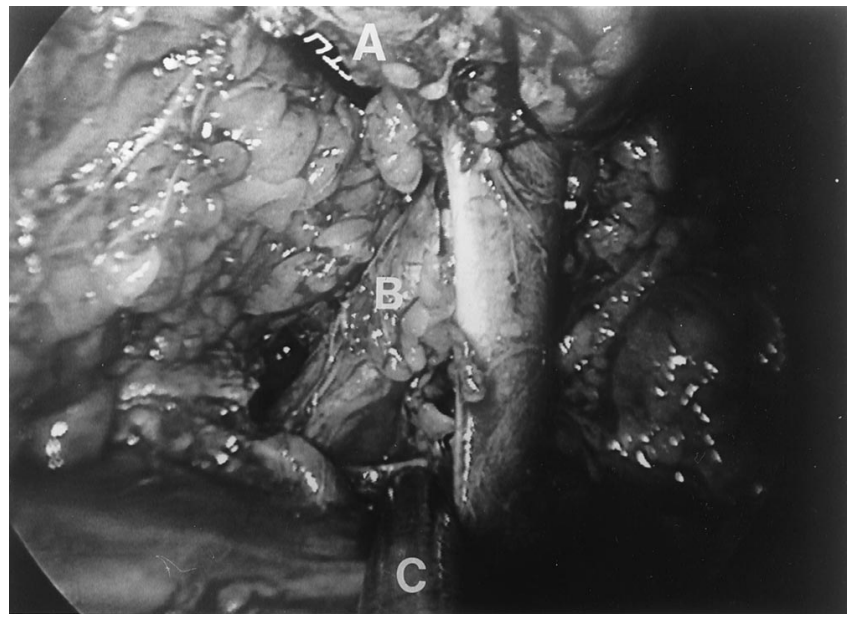

Figure 5. Renal hilum dissection elevating the kidney with harmonic scalpel (A) and preparing to staple the renal artery (B) with an articulating stapler $(\mathrm{C})$.

$12 \%)$. Most right kidneys had solitary arteries (82/97; 82\%), followed by 14 double renal arteries and a single case of triple renal artery graft that went unrecognized before surgery.

The mean surgical time was $235.0 \pm 66.7$ minutes (range 100-600) (Table 3). The mean fluid administration was $5.2 \pm$ 1.1 L crystalloid (range 3.2-8.0). Estimated blood loss was limited, with a mean of $139.2 \pm 165.8 \mathrm{~mL}$ (range 20-1,200). Three patients (3\%) required conversion to an open procedure for bleeding $(\mathrm{n}=1)$, renal artery injury $(\mathrm{n}=1)$, and uncertain vascular anatomy $(\mathrm{n}=1)$. Warm ischemic time was short at $238 \pm 112$ seconds (range 100-600). Most patients returned to regular diet by 18 to 24 hours $(50 / 97 ; 50 \%) ; 33$ patients (33\%) returned to diet within 18 hours. Four patients (4\%) had prolonged ileus and could not return to diet until almost 36 hours.

The length of hospital stay also varied. Most patients were discharged between 24 and 48 hours $(51 / 97 ; 51 \%)$. A few patients were discharged before 24 hours $(12 / 97 ; 12 \%)$. Eighteen patients (18\%) were discharged between 48 and 72 hours. Only 13 patients (13\%) were discharged after 72 hours.

Complications occurred in 14 of 97 (14\%) patients, with the three conversions considered complications (Table 4).

Table 1. INDICATIONS FOR RIGHT NEPHRECTOMY

\begin{tabular}{ll}
\hline - Multiple Left Arteries & $(n=59)$ \\
- Right Renal Cysts & $(n=8)$ \\
- Smaller Right Kidney & $(n=5)$ \\
- Retro-aortic Renal Vein & $(n=6)$ \\
- Right Renal Artery Stenosis & $(n=1)$ \\
Early Left Renal Bifurcation & $(n=2)$ \\
- Ectopic Kidney & $(n=1)$ \\
- Unknown & $(n=14)$ \\
\hline
\end{tabular}


Table 2. PATIENT DEMOGRAPHICS

\begin{tabular}{lcc}
\hline & Mean & Range \\
\hline M:F & $45: 52$ & \\
LRD:LURD & $73: 24$ & \\
Age (y) & $40.3 \pm 12.5$ & $18-69$ \\
Weight (lbs) & $171.0 \pm 37.1$ & $110-280$ \\
\hline
\end{tabular}

LRD:LURD, living related donor:living unrelated donor

There were three accessory upper pole injuries with minimal areas of ischemia noted. Two liver lacerations were produced while retracting the liver; neither required open conversion or surgical repair. Two postoperative infections occurred: a urinary infection and postoperative pneumonia. A case of bleeding from a vena caval suture line occurred, requiring laparoscopic homeostasis. One patient had a minor pulmonary embolus despite the use of pneumatic compression devices. Two grafts were lost in the initial 30 patients. These graft losses were the result of early venous thrombosis and in one patient was due to the kinking of a saphenous extension graft.

Mean serum creatinine levels were $5.2 \pm 2.9 \mathrm{~g} / \mathrm{dL}$ at 1 day and $2.1 \pm 1.7 \mathrm{~g} / \mathrm{dL}$ on day 7 (Fig. 6). This was slightly higher than in the previously reported open donor and left laparoscopic donor nephrectomy grafts harvested at the University of Maryland ( $P=\mathrm{NS}$ ). By 30 days, the difference of mean creatinine values among all groups was negligible. At 180 and 365 days, there was a small but nonsignificant trend toward an increased creatinine level in the right donor group.

\section{DISCUSSION}

The advantages of laparoscopic donor nephrectomy over open donor nephrectomy have been previously well established by multiple groups. ${ }^{4-710}$ Live-donor transplantation has notable benefits over cadaveric organs, particularly decreased rates of acute tubular necrosis and improved longterm graft survival. Recent data from the University of Maryland showed that laparoscopic donor nephrectomy does indeed increase organ donation, especially in whites and recipients older than 55 years of age. ${ }^{1}$ This donor method has also made an impact in the black population,

Table 3. OPERATIVE DEMOGRAPHICS

\begin{tabular}{lcc}
\hline & Mean & Range \\
\hline Fluids (L) & $5.2 \pm 1.1$ & $3.2-8.0$ \\
EBL (cc) & $139.2 \pm 165.8$ & $20-1200$ \\
Operative time (min) & $235.0 \pm 66.7$ & $100-446$ \\
Warm ischemic time (sec) & $238 \pm 112$ & $100-600$ \\
\hline
\end{tabular}

\section{Table 4. INCIDENCE OF DONOR} COMPLICATIONS

- Conversion to open procedure

- Upper pole arteries injured

- Need for arterial reconstruction

- Liver lacerations

- Recipient graft loss (Renal vein thrombosis)

- Infection (lung, urine)

- Bleeding vena cava

- Pulmonary embolus

$3 / 96(3 \%)$

$3 / 96(3 \%)$

$3 / 96(3 \%)$

$2 / 96(2 \%)$

$2 / 96(2 \%)$

$2 / 96(2 \%)$

$1 / 96(1 \%)$

$1 / 96(1 \%)$

increasing the donor pool. The left kidney is favored for laparoscopic nephrectomy because it provides a graft with a longer renal vein. ${ }^{4,5}$ Traditionally, right open donor nephrectomy is chosen when the left kidney has multiple renal arteries or veins or other vascular anomalies. The right is also chosen when it is an overall smaller organ or when a cystic mass is present. Harvesting the right kidney often shortens this innately short renal vein, increasing the complexity of the recipient procedure. When right-sided laparoscopic donor nephrectomy was introduced, the principle concern over renal vein division was the vascular staple line, which would shorten this already compromised renal vein.

The Johns Hopkins group was one of the first to perform right-sided laparoscopic donor nephrectomy. ${ }^{8}$ During their initial experience, three of eight (37.8\%) procedures were complicated by renal vein thrombosis. Two of these grafts had duplicated renal vein systems, and this was thought to contribute to the early thrombosis. This led to their adapting their technique by relocating their extraction excision and performing open division of the right renal vein, lengthening the right renal vein by panel graft from the recipient's saphenous vein, and changing the stapler port to allow parallel division of the renal vein from the vena cava. Subsequent to these changes, the Johns Hopkins group has

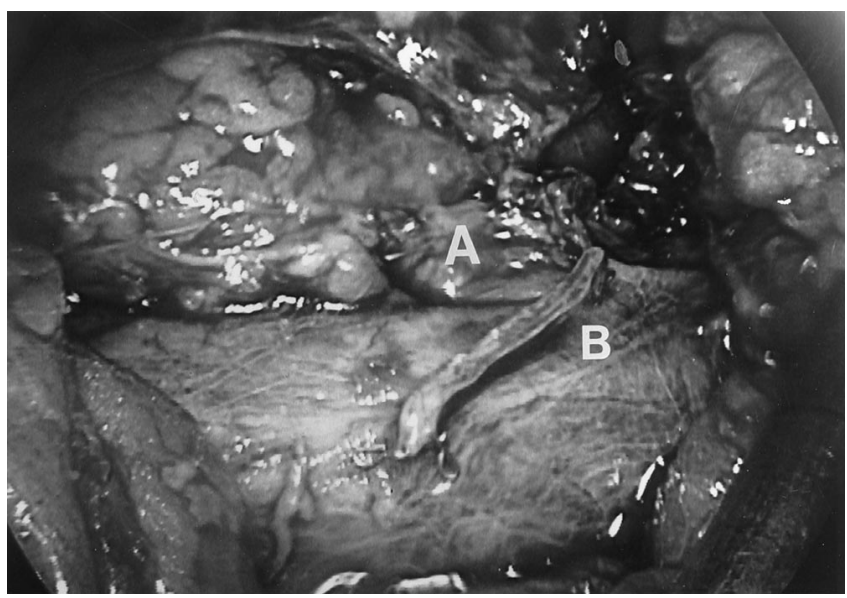

Figure 6. Vessels after the graft is removed demonstrating the stapled renal artery (A) and vena cava (B). 


\section{Mean Recipient Creatinine by Donor Operation}

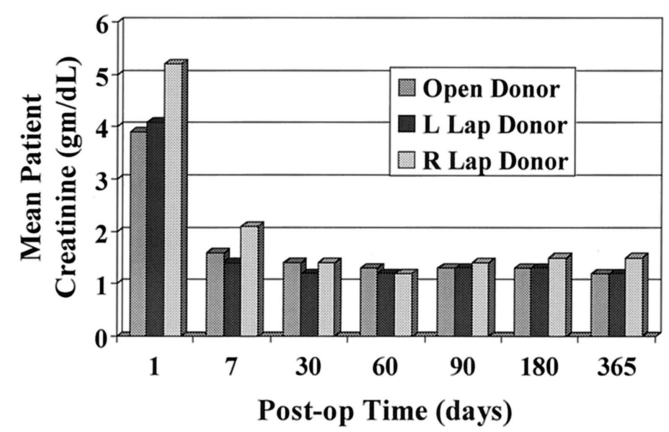

Figure 7. Comparison of short- and long-term serum creatinine values for right-sided laparoscopic, open, and left-sided laparoscopic donor recipients.

proceeded with nine additional right-sided laparoscopic donors without graft loss. The other group that has reported preliminary results is the Penn State group, who performed a majority of right donor nephrectomies with $8 / 15(60 \%)$ without delayed graft function or acute tubular necrosis, with a mean serum creatinine value of $1.1 \pm 0.1 \mathrm{~g} / \mathrm{dL}$ at 3 months.

In our series we report two early graft losses that resulted from short renal vein thrombosis. Both grafts were lost early in this experience, with one graft implanted with a saphenous vein extension graft that was thought to have torsed during the recipient procedure. Despite early recognition and thrombectomy, this graft was subsequently lost to rethrombosis. In the second case, the donor surgeon failed to recognize how short the right renal vein was $(5 \mathrm{~mm})$. After division with a stapler the length of common vein, $3 \mathrm{~mm}$ in length remained with a thin posterior wall. Once both anastomoses had been completed during implantation, the kidney was rotated to inspect the venous anastomosis, which avulsed. Subsequent repair of the anastomotic dehiscence was complicated by venous thrombosis. These early experiences stress that both the donor and recipient operation must be approached with equal caution. Preoperative imaging that shows the length and morphology of the right renal vein should be employed when the right kidney is being considered for harvest. When the vein is less than $15 \mathrm{~mm}$ in length, the right side is being considered for harvest. When the vein is less than $15 \mathrm{~mm}$ in length, the right side is best avoided, or the reasons for choosing it should be reviewed. For, example, if there are two arteries on the left (the most common reason), it may be better to plan a dual artery graft than chance a problem with a shorter vein. The donor operation must focus on meticulous, complete mobilization of the right renal vein, division parallel to the vena cava, with the vein and vena cava drawn well into the stapler. This will maximize the length of the vein without significantly narrowing the cava at that point. During the recipient oper- ation the venous anastomosis should be performed in a tension-free manner, which may require extensive mobilization of the external iliac vein by division of the internal iliac vein or placement of the graft in an intra-abdominal position.

Two unique complications are presented with the description of right-sided laparoscopic donor nephrectomy. The first is the inadvertent injury to the liver during the retraction of the liver for exposure and dissection of the superior pole of the kidney. This usually results when the instrument retracting the liver is not visualized by the camera. Multiple instruments have been used for retraction including a laparoscopic Kitner, balloon retractor, or metallic triangle retractor. The simplest method of liver retraction used by one of the authors is placing a $5 \mathrm{~mm}$ port just below the xiphoid process, and elevating the liver with a toothed grasper and clamping it to the diaphragm where it will hold the liver until the end of the procedure. Another unusual but potentially devastating complication is bleeding from the vena cava. On one occasion in this series, the stapler had been pushed firmly against the lower edge of the vein when fired, resulting in localized bleeding due to a deficit of staples at that point. After a period of pressure with gauze sponge, a single stitch was sufficient to arrest the bleeding. Extensive failure of the staple line after the vein has been divided with the stapler's knife is an extremely hazardous event. Prevention is the best strategy. For that reason some authors advocate staplers which do not divide when fired $(12 \mathrm{~mm}$ endo-TA with vascular 2.5 length staples, TYCO Healthcare, Norwalk CT). After application of the stapler, the staple line is carefully inspected for completeness, a partial cut of the vessel made with scissors, the absence of back bleeding confirmed and the cut completed. The management of small accessory upper pole arteries has been experienced in left and right kidneys. When small accessory poles are encountered, meticulous detail must be paid to prevent injury. However, removal of the adrenal gland with redundant arteries may result in sacrificing a small redundant upper pole artery. With the use of pre-operative angiograms you can often discern the involved branch and confirm that the risk parenchymal area has redundant renal artery flow.

Long-term renal function has been assessed by serum creatinines in the postoperative period. At 1 and 7 days the right-sided laparoscopic donor grafts had a slightly higher creatinine when compared to previously presented data from the follow-up paper from the University of Maryland with open and left-sided laparoscopic donor grafts. By the 30-day mark mean serum creatinines were identical, with only a small but statistically nonsignificant stable increase in mean serum creatinines in the right-sided donors by 6 and 12 months.

In summary, right-sided laparoscopic donor nephrectomy carries an initially steep learning curve that should be approached with respect. Early complications were experienced and identified by several groups, as was the case with 
in left laparoscopic donor nephrectomy. We recommend that the right renal vein be extensively mobilized and divided parallel to the vena cava, after drawing a little of the caval wall into the stapler jaws to increase renal vein length. Liver injury can be avoided with the self-retaining technique described. This study should serve to stress the importance of the implantation operation and insure adequate tension-free anastomosis, whether this requires mobilizing the external iliac vein, placing the graft on the vena cava, or even inverting the graft where the inferior pole of the kidney is placed in the upper abdomen to sit better against the iliac vein or the recipient vena cava. With attention to these varying issues, the performance of right-sided laparoscopic donor nephrectomy can be accomplished safely with excellent long-term graft function.

\section{References}

1. Schweitzer EJ, Wilson J, Jacobs S, et al. Increased rates of donation with laparoscopic donor nephrectomy. Ann Surg 2000; 232:696-703.

2. Morris PJ. Results of renal transplantation. Philadelphia: WB Saunders; 1994:504-523.

3. Groth CG, Fehrmman A, Ringden O, et al. Related donor kidney transplantation is the best form of treatment for uremia. Transplant Proc 1987; 19:2278-2279.

4. Jacobs SC, Cho E, Dunkin BJ, et al. Laparoscopic live donor nephrectomy: the University of Maryland 3-year experience. J Urol 2000; 164:1494-1499.

5. Ratner LE, Montgomery RA, Kavoussi LR. Laparoscopic live donor nephrectomy: the four-year Johns Hopkins University experience. Nephrol Dial Transplant 1999; 14:2090-2093.

6. Philosphe B, Kuo PC, Schweitzer EJ, et al. Laparoscopic versus open donor: comparing ureteral complications in the recipient and improving the laparoscopic technique. Transplantation 1999; 68:497-502.

7. Flowers JL, Jacobs S, Cho E, et al. Comparison of open and laparoscopic live donor nephrectomy. Transplantation 1997; 226:483-490.

8. Mandal AK, Kalligonis AN, Cohen C, et al. Should the right kidney be used in laparoscopic live donor nephrectomy? Transplantation 2000; 69:S403.

9. Arenas J, Gupta M, et al. Initial program experience with right laparoscopic donor nephrectomy. Transplantation 2000; 69:S335.

10. Slakey DP, Wood JC, Hender D, et al. Laparoscopic living donor nephrectomy: advantages of the hand-assisted method. Transplantation 1999; 68:581-583.

\section{Discussion}

DR. ARNOLD G. DIETHELM (Birmingham, Alabama): I'd like to thank Dr. Fischer and Dr. Buell for asking me to comment on their paper. This is an important technical consideration, and I believe the results speak for themselves.

The real question they have asked and answered: is there a greater risk to the right donor nephrectomy than the left. Now I think there are several comments I'd like to make and then ask some technical questions of Dr. Buell, because the comments regarding technique, I believe, are critical to a successful right nephrectomy open or closed.

First of all, the mobilization of the cava is critical in terms of the lumbar veins. You need to be sure you divide the lumbar veins adjacent to the renal artery. And my question is, have you had any difficulty in the management of those renal veins?

The second is, the right renal vein is very short. And the right renal vein substance, the thickness, is not great at all. The best solution for a right nephrectomy for purposes of transplantation is to take 2 or possibly $3 \mathrm{~mm}$ of the vena cava, and that will, first, give you a wider diameter of the renal vein, and second, gives you a little more substance to sew to.

So, Dr. Buell, can you do this with laparoscopic nephrectomy?

It is important how you place a stapler or a clamp on the collar portion of the cava at the entrance of the renal vein, and you need to take a slight indentation on the cava in order to achieve that portion of the cava that will go with the renal vein to make the anastomosis easier. Can you do this satisfactorily with the endoscopic technique?

Last, if one has an insufficient right renal vein upon removal of the kidney, in my experience, the best solution is to cool the kidney, put it on the back table, get a vein allograft, do an ex-vivo anastomosis of that allograft as an extension graft and then get away from the problems associated with the short renal vein.

Now the short renal vein is particularly a problem in the patient that is slightly obese, particularly a male, and seemingly more difficult with a black male, where the pelvis is narrow and deep. Therefore, what I would really like Dr. Buell to answer in addition to the other questions, is there any anatomy that you would shy away from with a laparoscopic nephrectomy?

Second, how do you manage double renal veins? Do you staple one, take the other out? Do you take them both out together? Or does it depend upon the distance between the two? How would you manage triple renal arteries?

And the last question is, what do you do in the rare occasion when there is a right renal artery coming from the common iliac or, very rarely, the external iliac?

I think the paper is an important contribution. It clears up some uncertainties, and I would like to thank them for asking me to comment.

Thank you.

VICE PRESIDENT LAWRENCE: Is there anyone else who would like to comment on this interesting paper?

It seems to show, incidentally, that - and this may be in contrast to what Dr. Aust told us today - technique may be important to this.

DR. JOSEPH F. BUELL (Cincinnati, Ohio): Thank you, Dr. Lawrence, Dr. Townsend, and the Society for allowing us to present this data. Obviously, there have always been a lot of naysayers to laparoscopic surgery, first in laparoscopic cholecystectomy, then secondarily laparoscopic Nissen fundoplication. Despite this, we have seen a higher referral rate of patients for cholecystectomy and for Nissen fundoplications.

Laparoscopic kidney donation has been shown by Dr. Bartlett to actually increase the amount of grafts available for people waiting for kidney transplantation and decreasing the death on the waiting list for these unfortunate individuals.

In the performance of right donor nephrectomy, unfortunate series have demonstrated a high incidence of vascular complications, namely, venous thrombosis due to the short vascular vein that they are left with. This has also been seen in patients with left laparoscopic nephrectomy that have retro-caval veins as well. Thus, the problem is short renal veins.

I'd like to address the comments from Dr. Diethelm about mobilization of the vena cava. As he has so eloquently noted, the lumbar veins are problematic in the mobilization of the right kidney. These veins are best dealt with by mobilizing the kidney forward, allowing exposure of the posterior aspect of the vena cava. These veins are extremely short and enter the retroperitoneum and can be dealt with either by the harmonic scalpel, if they are less than $3 \mathrm{~mm}$ in diameter, or they can be clipped with a right-angle clip applier. These allow further extension posterior to the vena cava, giving us a long length of renal artery.

Secondly, how do we deal with our renal vein to get it to be a little bit longer? Well, some individuals have adopted the use of an additional port to elevate the kidney, or, as our group has accepted, the use of a hand-assist laparoscopic device, or the ability to place the operative surgeon's hand in the abdomen and pull the kidney back. This allows the vascular stapler to be brought through the $12 \mathrm{~mm}$ port and to place it in a parallel dimension with the vena cava. This way, the actual renal vein and some of the vena cava can be everted out, and you can get the stapler across the vena cava itself, taking a small amount of the better tissue of the vena cava to suture to.

What about panel grafts? Well, the Hopkins group evaluated their initial experience where they had problems. They did two things. They did open 
ligation of the renal vein, which made this much easier, and they also advocated the use of panel grafts as was suggested. And since that time they have had no vascular complications or thromboses during their subsequent experience.

The people that we look at or have problems with are males of higher weight. The highest rate that individuals have attempted to resect are 290 pounds. Again, with the hand-assist device, I think this makes it an easier option to elevate and get a longer length of their renal vein in these heavier patients.

Is there anatomy that we would not approach? Three renal arteries on the right side. We would not do a horseshoe kidney, despite several individuals performing this with good graft function subsequent.

How do we manage double or triple arteries? Well, on the left or the right side, our hopes are that they would be in close proximity, which would allow the vascular staple to go across both renal arteries or all three renal arteries at the same time. One paper by Paul Kuo noted that at 3 arteries or 4 arteries, your creatinine at a 3-month interval and, eventually, at a year interval are higher and you have a higher incidence of ATN in these patients. Thus, we try to avoid these patients.

Lastly can you manage a renal artery arising from the iliac artery? If you have a renal artery arising from the iliac artery, I believe the hand-assist device is most beneficial in this situation. The reason why we advocate the hand-assist device is it allows better mobilization of the ureter and prevents ureteral stenosis because there is no manipulation of the artery down in the area of the distal ureter. The other benefit is that it allows full direct visualization of the iliac artery, and in this case we would be able to directly divide the artery through the hand-assist device and would make this feasible.

Thank you for allowing us to present this data. 